Full Length Article

\title{
Cortical bone mapping improves finite element strain prediction accuracy at the proximal femur
}

\author{
Enrico Schileo $^{\mathrm{a}, *}$, Jonathan Pitocchi ${ }^{\mathrm{b}, \mathrm{c}, \mathrm{d}}$, Cristina Falcinelli ${ }^{\mathrm{e}}$, Fulvia Taddei ${ }^{\mathrm{a}}$ \\ ${ }^{a}$ Bioengineering and Computing Laboratory, IRCCS Istituto Ortopedico Rizzoli, Bologna, Italy \\ ${ }^{\mathrm{b}}$ Materialise N.V., Heverlee, Belgium \\ ${ }^{\mathrm{c}}$ Multiscale in Mechanical and Biological Engineering (M2BE), University of Zaragoza, Zaragoza, Spain \\ ${ }^{\mathrm{d}}$ Biomechanics Section, KU Leuven, Leuven, Belgium \\ ${ }^{\mathrm{e}}$ Department of Engineering, Campus Bio-Medico University of Rome, Italy
}

A R T I C L E I N F O

\section{Keywords:}

Cortical bone

Finite elements

Computed tomography

Cortical bone mapping

Validation

Subject-specific Modelling

Proximal femur

Density-elasticity relationship

Optimisation

\begin{abstract}
A B S T R A C T
Despite evidence of the biomechanical role of cortical bone, current state of the art finite element models of the proximal femur built from clinical CT data lack a subject-specific representation of the bone cortex.

Our main research hypothesis is that the subject-specific modelling of cortical bone layer from CT images, through a deconvolution procedure known as Cortical Bone Mapping (CBM, validated for cortical thickness and density estimates) can improve the accuracy of CT-based FE models of the proximal femur, currently limited by partial volume artefacts. Our secondary hypothesis is that a careful choice of cortical-specific density-elasticity relationship may improve model accuracy.

We therefore:

(i) implemented a procedure to include subject-specific CBM estimates of both cortical thickness and density in CT-based FE models.

(ii) defined alternative models that included CBM estimates and featured a cortical-specific or an independently optimised density-elasticity relationship.

(iii) tested our hypotheses in terms of elastic strain estimates and failure load and location prediction, by comparing with a published cohort of 14 femurs, where strain and strength in stance and fall loading configuration were experimentally measured, and estimated through reference FE models that did not explicitly model the cortical compartment.

Our findings support the main hypothesis: an explicit modelling of the proximal femur cortical bone layer including CBM estimates of cortical bone thickness and density increased the FE strains prediction, mostly by reducing peak errors (average error reduced by 30\%, maximum error and 95th percentile of error distribution halved) and especially when focusing on the femoral neck locations (all error metrics at least halved). We instead rejected the secondary hypothesis: changes in cortical density-elasticity relationship could not improve validation performances. From these improved baseline strain estimates, further work is needed to achieve accurate strength predictions, as models incorporating cortical thickness and density produced worse estimates of failure load and equivalent estimates of failure location when compared to reference models.

In summary, we recommend including local estimates of cortical thickness and density in FE models to estimate bone strains in physiological conditions, and especially when designing exercise studies to promote bone strength.
\end{abstract}

\section{Introduction}

There is growing consideration for the role of cortical bone compartment in determining bone biomechanics, and ultimately bone strength $[1,2]$. This is particularly true at the proximal femur, one of the most relevant fracture sites. Cortical thinning and increased cortical porosity at the femoral neck are main contributors to bone strength decline, and their combination may directly explain fracture events, especially in women [3]. Consequently, counterbalancing aging-related cortical bone loss has become a target for bone drugs [4], and exercise protocols [5].

Under load, cortical compartment takes a larger load-share than

\footnotetext{
* Corresponding author at: Bioengineering and Computing Laboratory, IRCCS Istituto Ortopedico Rizzoli, Via di Barbiano, 1/10, 40136 Bologna, Italy.

E-mail address: enrico.schileo@ior.it (E. Schileo).
} 
trabecular compartment [6,7]. Cortical bone predominance in determining femoral strength has been indirectly assessed by [8] by achieving a good validation of cortical-only FE models vs. experiments conducted on intact femurs; and directly by [9] by testing intact vs. contralateral trabecular-excavated femurs, finding strength differences as low as $7 \%$ on average. In partial contradiction to these macro-level findings, microscale FE models led to the hypothesis that in the proximal portion of the femoral neck trabecular bone takes a larger load share than cortical, but moving distally cortical bone progressively bears more load [10]. In brief, the importance of cortical bone contribution to overall proximal femur biomechanics is beyond doubt.

Despite its importance, cortical bone is often not explicitly modelled in CT-based models of the proximal femur. And, when modelled, it is generically defined through a priori assumptions on its thickness and elasticity, often assumed homogeneous $[8,11]$. This is because an accurate subject-specific representation of thin bone cortices from clinical CT is hindered by image blurring, mostly due to partial volume effect (PVE) [12], which makes cortices appear larger and less dense than they are. There is evidence that PVE in CT images influences FE results $[13,14]$, and in a past study we found that peak errors in FE predictions against experimental strain gauge measurements occurred, most likely due to PVE, at sites of thin cortical bone shell in the proximal femur [15].

Past efforts (including ours) in reducing PVE effect when generating FE models of bone segments from CT images, have in fact led to global improvements in models accuracy [16-18] but could not resolve local PVE issues, possibly because they do not permit an explicit, accurate subject specific modelling of the cortical bone compartment.

Recently, Treece et al. developed cortical bone mapping (CBM), which estimates cortical parameters from CT images by way of a deconvolution algorithm, producing local estimates of both cortical thickness (i.e. permitting to identify both periosteal and endosteal bone cortex boundaries) and density, validated against HRpQCT [19]. CBMderived parameters have already shown an independent contribution to the identification of fractures within a large clinical study [20], and have helped explain the mechanisms of femoral fracture due to focal osteoporosis [21]. However, CBM has been so far mostly used to conduct morphological and densitometric analyses. Two very recent studies [22,23] attempted the inclusion of CBM estimates into FE models, but were apparently limited to cortical thickness.

Our main research hypothesis is that the inclusion of CBM-derived, subject-specific estimates of cortical thickness and cortical density into CT-based FE models of the proximal femur can improve the accuracy of strain and strength predictions. In this study we describe the implementation of CBM density and thickness estimates into FE models, and report the validation of CBM-augmented FE models using specimens, images, and reference models from a previous study [18].

Our secondary hypothesis is that the density-elasticity relationship used to assign CT-based inhomogeneous Young's moduli in the previous reference study, derived from trabecular specimens only [24], might not be adequate when explicitly modelling the bone cortex. In addition, several recent studies supported for the femur the use of "optimised" density-elasticity relationships, either individual-based [25], stochastically derived [26], or tuned to large data samples [27]. Therefore, we tested whether the use of a cortical-specific or of an optimised densityelasticity relationship could improve the validation results of CBMaugmented FE models.

\section{Methods}

We developed a workflow to include subject-specific estimates of cortical thickness and density obtained from CBM in CT-based FE modelling of the proximal femur; and we validated it using an already published ex-vivo dataset.

In fact, we used images, experimental measurements, and we compared to FE-derived results of a previous validation study in which we tested a CT deblurring algorithm [18] on 14 human femora. The essentials of the experimental tests were the following:

- 10 specimens were instrumented with 12 triaxial strain gauges around the femoral head-neck junction, neck and metaphysis. Each strain gauge measured three strain components that were elaborated into two principal components of the surface strain. These specimens were tested in the elastic range, in 6 loading configurations aimed to mimic single leg stance (10 specimens), and 12 loading configurations aimed to mimic sideways falling (7 specimens). The total number of valid data points measured was 3314 (142 data point were either not acquired due to hardware problems or discarded due to measurement/signal elaboration issue)

- all 14 specimens were loaded up to failure (7 in single leg stance, 7 in sideways falling configuration).

Details of the experimental setup were originally described in previous works for both stance [28] and fall [29] loading conditions. Specimens' details are reported as Supplementary Material Table I.

In the present study, we relied on the CT images of the 14 human femora to build new FE models with cortical information (hereinafter referred to as CBM models). These CBM models were then loaded and constrained to replicate the boundary conditions present in the experimental tests. Finally, we compared the results of CBM models (elastic strains and strength to failure) with (i) the experimental results and (ii) the FE results obtained without cortical bone information, fully reported in [18] and hereinafter referred to as reference FE results. The numerical procedures used to obtain the reference FE results have been described in previous works, for elastic strain [30,31] and for failure [32].

The novel contribution of the present study includes constructing FE models with cortical information (CBM models), exploring the use of different density-elasticity relationships, calculating the strain and the failure load for the CBM models, and comparing them to experimental results and to reference FE results.

\subsection{From CT images to FE meshes using CBM estimates of cortical thickness (Fig. 1)}

We performed CBM using the freeware Stradwin (version 5.3, University of Cambridge, UK). Firstly, we manually segmented the proximal femur to have an initial estimate of the periosteal surface, used as input for the CBM. In brief, the CBM algorithm (defined in the most recent Stradwin version as CBMv2) uses a profile analysis of actual $\mathrm{HU}$ values along the local surface normal direction to obtain, assuming an ideal rectangular profile shape, deconvolved cortical thickness and density through non-linear optimisation [19]. The proximal femur surface is sampled locally at 5000-10000 surface points, then CBMbased cortical thickness estimates at these points are used to generate by triangulation the periosteal and endocortical surface, thus identifying the cortical bone layer through two tessellated surfaces featuring local CBM density estimates at their nodes. CT images had a pixel size ranging from 0.48 to $0.66 \mathrm{~mm}$, and an out-of-plane reconstruction spacing ranging from 1 to $2 \mathrm{~mm}$ (of note, CBM includes a different formulation of blurring perpendicular to the CT slicing plane).

To generate the FE models using CBM, we first obtained B-spline representations of surfaces (IGES models) (Geomagic Studio, v. 7, Raindrop Geomagic, Inc., USA). From the IGES models, unstructured FE-meshes were generated using an advancing-front algorithm with 10node tetrahedra both for the cortical layer (average element edge length $1 \mathrm{~mm}$ ) and the trabecular compartment (element edge length $1 \mathrm{~mm}$ at cortical interface and extending to $3 \mathrm{~mm}$ inwards) (Hypermesh 13.0, Altair Engineering Inc., USA). On average, the FE models of the proximal femora consisted of around 500 thousand elements and 750 thousand nodes, almost equally subdivided between the cortical and trabecular compartments. 




Fig. 1. The CBM model workflow consists of: tentative manual segmentation (A), estimation of the cortical boundaries with Stradwin (version 5.3, University of Cambridge, UK) (B), generation of two distinct FE models for the cortical and trabecular compartments (C).

\subsection{Material properties mapping using CBM estimates of cortical density}

To derive inhomogeneous elastic bone properties from HU values we applied the same relationships described in the reference study [18]. In brief we:

- obtained radiological density $\left(\rho_{Q C T}\right)$ from HU values through the dataset-specific, phantom-derived CT densitometric calibration;

- converted $\rho_{Q C T}$ to ash density $\left(\rho_{a s h}\right)$ using the linear correction proposed in [31];

- converted $\rho_{a s h}$ to apparent wet density $\left(\rho_{a p p}\right)$ using the ratio $\left(\rho_{a s h} /\right.$ $\left.\rho_{\text {app }}=0.6\right)[31]$;

- calculated the elastic modulus $E$ using the $E=6.850 * \rho_{a p p}{ }^{1.49}$ density-elasticity relationship [24], with E in GPa and $\rho_{\text {app }}$ in $\mathrm{g} / \mathrm{cm}^{3}$. Poisson's ratio was set to 0.3 .

In the trabecular compartment, these relationships were applied to all CT voxels falling (even partly) within the FE model volume, and then elastic properties were mapped from cubic CT voxels to each tetrahedral element of the FE mesh using the classical Bonemat algorithm (freeware at www.bonemat.org) that performs a numerical integration of voxel-wise properties into each element's volume.

For the cortical layer, we developed a specialised Bonemat version (now included in the freeware available at www.bonemat.org). This specialised Bonemat uses as input the cortical density values sampled at the outer surface locations of the cortical object. Then, for each element of the cortical FE mesh it performs a numerical integration of the cortical intensity estimates from CBM. The numerical integration happens with the same principles and integration scheme (based on element Gauss points) of that conducted on CT voxels for the elements of the trabecular compartment, described in earlier studies [33,34]. Since
CBM vertices may be more sparsely distributed than FE elements, if no CBM vertex is included in the volume domain of an integration point, the nearest CBM vertex outside the element is searched. It is worth recalling that in CBM surface reconstruction, periosteal and endosteal boundaries are represented by paired triangles (whose paired endosteal and periosteal vertices represent the paths of the HU profile analysis), and that CBMv2 implementation assumes constant density through the cortex. Therefore, each two corresponding endosteal and periosteal vertices share the same density estimate.

Ultimately, we applied a double threshold to the estimated values of $\mathrm{E}$ of the cortical layer to remove possible outliers generated by the nonlinear optimisation of the CBM algorithm. Such optimisation can fail at some surface points, thus generating unreliable density estimations. We defined a specimen-specific maximum threshold, by seeking for the maximum $\mathrm{HU}$ value found in cortical bone $\left(\mathrm{HU}_{\max }\right.$, typically found in compact cortical bone at the mid-diaphysis), then filtering the data to exclude all the values of cortical elastic modulus higher than the $\mathrm{E}_{\max }$ corresponding to the $\mathrm{HU}_{\max }$ according to the chosen relationships. Then, we defined for all datasets a global minimum threshold of $4.5 \mathrm{GPa}$, i.e. the lowest measured cortical bone Young's modulus in a wide experimental campaign including also child bone [35]. As a consequence, an average of $1 \%$ of cortical elements (min: $0 \%$, max: $3 \%$ ) was filtered because exceeding the high threshold, and an average of $3 \%$ cortical elements (min: $0 \%$, max: $8 \%$ ) because falling below to the low threshold. The difference in Young's modulus at surface elements between CBM and reference models is manifest (Fig. 2).

\subsection{Alternative material properties mapping using CBM estimates of cortical density}

To verify the secondary hypothesis of our study, we tested
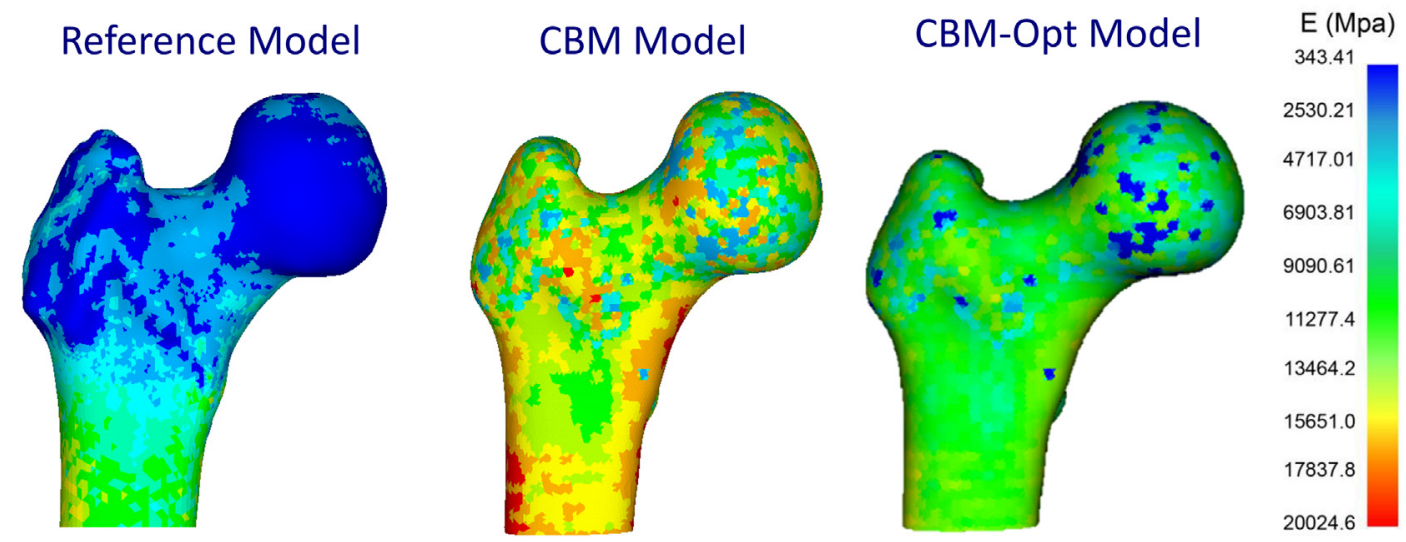

Fig. 2. Young's Modulus mapped directly from CT values on the FE reference Model (left), and from deconvolved estimates of cortical intensity using the standard material mapping procedure (CBM Model, center), or the optimised density-elasticity relationship (CBM-Opt model, right). 
alternative material mapping solutions.

\subsubsection{Upper threshold to trabecular bone properties (CBM-Tb.Limit models)}

One possible issue related to CBM is partial double-counting of cortical density. When sampling the original CT volume to obtain material properties for the trabecular compartment of the FE model, data very close to the cortex may contain some residual blur from the cortex itself, which has already been accounted for within the cortical data, and should not be double-counted in the trabecular data. We actually verified that trabecular compartments of CBM models contained elements with Young's modulus up to $18 \mathrm{GPa}$. We thus applied to the trabecular bone an upper threshold equal to the lower threshold applied for cortical bone $\left(4.5 \mathrm{GPa}\right.$, corresponding to a $\rho_{\text {app }}$ of $0.8 \mathrm{~g} / \mathrm{cm}^{3}$ according to [24]). On average, a notable $16 \%$ of the trabecular elements were filtered. As we believe this filter corrected a systematic bias of the CBM FE models, we retained it in all the alternative material mapping strategies described in the following paragraphs.

\subsubsection{Cortical-specific density elasticity relationship (CBM-Cort models)}

We conducted a systematic review of the literature to identify all studies investigating density-elasticity relationships of human cortical bone (see Appendix 1, with a Table summarising all studies and a graph plotting all relationships viable for use in FE models, after harmonisation of density measures to $\rho_{\text {app }}$ ). In brief, contrarily to what happened for trabecular bone (over 20 relationships reviewed back in 2008 [36]), only 10 works spanning 40 years of research were dedicated to cortical bone (Appendix 1, Table A.1). Of note, testing methodologies ranged from ultrasound techniques to three-point bending, to different implementations of compressive and tensile tests. Among these studies, we progressively excluded:

- one study failing to explicitly report equations [37],

- two studies reporting separated tension/compression responses (inapplicable to standard FE models) [38,39]

- the two linear relationships $[40,41]$ that were evidently thought for use only in the rather narrow range of measured density, because when extended below this range (but at values still viable for cortical bone) they produced unrealistic Young's moduli, i.e. negative moduli at $\rho_{\text {app }}$ of around $1 \mathrm{~g} / \mathrm{cm}^{3}$;

- the two relationships [42,43] that although rather popular in FE studies, were derived from compressive tests not following a state of the art technique: according to a review [36], the platen technique in [43] is prone to errors, the confined compression in [42] may overestimate response of dense specimens

- one ultrasound-derived relationship [44] because no coefficient of determination was reported for the power law.

The two relationships left, $[35,45]$ showed similar trends, although derived from different techniques. We chose that reported by Ohman et al., $E=12.9 * \rho_{a s h}^{2 . o}$ because fit on a very large density range (much larger than in [45]), and showing a higher determination coefficient.

\subsubsection{Optimised density elasticity relationship (CBM-Opt models)}

Interestingly, after 30 years since the first CT-based FE models, a lot of effort is still put in the choice of density elasticity relationships which best fit the modelling purpose, [46-48]. Focusing on the femur, there is evidence from three different approaches [25-27] that optimisation of the density-elasticity relationship can improve the accuracy of $\mathrm{FE}$ models of bones. We excluded the optimisation attempt requiring the development of individual relationships [25] because it lacks the generality to be prospectively applicable in vivo. The other two studies implemented radically different approaches (direct optimisation of the parameters of a power density-elasticity law on FE validation results [27] vs. extraction of median values from a stochastic analysis of raw data from previous mechanical tests aimed at defining density-elasticity laws [26]) but interestingly came to quite similar results, somehow providing a mutual corroboration. We chose to use the relationship by Rezaei et al. [27], because it directly optimised FE results on a large set of femora (100). We thus implemented to the cortical compartment the relationship $E=11046{ }^{*} \rho_{a s h}{ }^{1.36}$ (both the trabecular and cortical thresholds above described were kept active), obtaining models that will be hereinafter called CBM-Opt models.

\subsubsection{Note about results of CBM-Tb.Limit and CBM-Cort models}

The use of an upper threshold to trabecular bone properties (CBMTb.Limit models) and its combination with a cortical-specific densityelasticity relationships (CBM-Cort models), tested first on two pilot specimens, did not induce significant changes in the validation with respect to CBM models. Results about CBM-Tb.Limit and CBM-Cort in these two pilot specimens are thus reported in Appendix 1. Results of CBM-Opt models are instead presented in the Results section.

\subsection{Boundary conditions}

Boundary conditions mimicked those of the experimental tests, following a spatial registration procedure. As only proximal femur cortical models were available, we adopted a cut-boundary displacement method to replicate the experimental distal constraint, as described in the reference study [18].

\subsection{Strain and strength estimates}

Linear FE analyses were performed (Ansys Inc., v. 15, USA), assuming at the surface a plane-stress state. Principal strains were computed adopting the same procedure described in [30]. Failure loads were calculated as described in [32], adopting a maximum principal strain criterion, with $0.73 \%$ strain limit in tension and $-1.04 \%$ strain limit in compression [49].

\subsection{Statistical analysis}

We used linear regression (reporting determination coefficient $\left(\mathrm{R}^{2}\right)$, slope, intercept, standard error of estimate (SEE)) and error metrics (root mean square error (RMSE) and peak error (Max. err), both absolute and in percentage with respect to the maximum measured value) to compare FE predicted strains and failure loads to experimental measurements.

All regression and error indicators were then compared to those obtained from the reference FE models. In particular, a $t$-test was used to statistically compare the slope of the regression equation of the CBM and CBM-Opt models to the reference ones, and a Wilcoxon test to compare the strain error distributions.

All analyses were performed with the free statistical software $\mathrm{R}$ (https://CRAN.R-project.org), considering the data pooled for stance and sideways fall loading conditions, and separated for the two loading configurations. These data pools were supported by statistical analysis on the difference between measured and FE predicted principal strains (Factorial ANOVA, $>0.9$ among loading configurations per each specimen, $>0.05$ among specimens). Additional pooling was performed to limit result analysis to the femoral neck region (all data from femoral neck strain gauges) and then to each strain gauge location, to understand whether CBM and CBM-Opt models effectively improved the highest, likely PVE-related, strain prediction errors.

\section{Results}

\subsection{Strain prediction accuracy}

\subsubsection{Pooled data}

We observed the biggest changes between CBM and reference models in error metrics (Table 1). CBM models, and to a lower extent 
Table 1

Strain accuracy indicators on pooled data for CBM, CBM-Opt and reference models.

\begin{tabular}{|c|c|c|c|c|c|c|c|c|c|}
\hline \multicolumn{10}{|l|}{ Whole femur } \\
\hline \multirow[t]{2}{*}{ Strain accuracy indicators } & \multicolumn{3}{|l|}{ Pooled } & \multicolumn{3}{|l|}{ Stance } & \multicolumn{3}{|c|}{ Sideways fall } \\
\hline & CBM & Ref. & CBM-Opt & CBM & Ref. & CBM-Opt & CBM & Ref & CBM-Opt \\
\hline $\mathrm{R}^{2}$ & 0.91 & 0.90 & 0.90 & 0.92 & 0.90 & 0.91 & 0.91 & 0.91 & 0.91 \\
\hline Slope & 0.83 & 1.17 & 1.01 & 0.89 & 1.09 & 1.08 & 0.82 & 1.19 & 0.99 \\
\hline Intercept $(\mu \varepsilon)$ & -2 & -5 & 0 & -10 & 5 & -12 & 3 & -11 & 7 \\
\hline $\operatorname{SEE}(\mu \varepsilon)$ & 150 & 221 & 191 & 110 & 152 & 146 & 169 & 255 & 213 \\
\hline $\operatorname{RMSE}(\mu \varepsilon)$ & 180 & 243 & 191 & 120 & 156 & 151 & 211 & 287 & 213 \\
\hline RMSE \% & 8.2 & 11.0 & 8.7 & 6.9 & 9.0 & 8.7 & 9.6 & 13.0 & 9.7 \\
\hline Max.err $(\mu \varepsilon)$ & 919 & 1819 & 1291 & 504 & 1298 & 715 & 919 & 1819 & 1291 \\
\hline Max.err \% & 41.7 & 82.6 & 58.6 & 29.1 & 74.8 & 41.2 & 41.7 & 82.6 & 58.6 \\
\hline
\end{tabular}

CBM-Opt models led to significant error reductions regardless of loading condition. Compared to reference:

- SEE values were reduced by approximately $30 \%$ in CBM $(-32 \%$ pooled data, $-28 \%$ stance, $-34 \%$ fall). In CBM-Opt: reduced by 14 in $\%$ pooled data and $16 \%$ in fall, but only by $4 \%$ in stance.

- RMSE values were reduced by approximately $25 \%$ in CBM $(-26 \%$ pooled data, $-23 \%$ stance, $-27 \%$ fall). In CBM-Opt: reduced by $21 \%$ in pooled data and $26 \%$ in fall, but only by $3 \%$ in stance. Of note, RMSE values for CBM and CBM-Opt were always below $10 \%$ of the maximum measured strain value.

- Peak errors (Max.err in Table 1) were at least halved in CBM ( $-49 \%$ for pooled data, $-61 \%$ for stance, $-49 \%$ for fall) and reduced by $30 \%$ also in CBM-Opt.

FE strain error distributions in CBM and reference models differed significantly in pooled data and stance data (Wilcoxon test, $p$-value $<$ $0.0001)$, but not in fall data $(p=0.18)$. Error distributions of CBM-Opt and reference models were less different (Wilcoxon $p=0.02$ for pooled data, 0.29 for stance, 0.10 for fall).

However, we confirmed CBM (and to a lower extent, CBM-Opt) improvements in peak errors by looking at the 95th percentiles of the FE strain error distributions. In CBM the 95th percentile was reduced by $24 \%$ (399 vs. $526 \mu \varepsilon$ ) in pooled data, by $27 \%$ ( 249 vs. $343 \mu \varepsilon$ ) in stance and by $31 \%$ ( 471 vs. $682 \mu \varepsilon$ ) in fall. In CBM-Opt, by $22 \%$ in pooled data, by just $1 \%$ in stance, and by $34 \%$ in fall.

As to metrics of regression with experimental data, we report:

- a substantial equivalence of CBM and CBM-Opt in terms of coefficient of determination $\left(\mathrm{R}^{2} \geq 0.90\right)$, which however did not improve significantly over reference models (Fig. 3, Table 1).

- a notable difference in regression slopes, with all models being mutually different $(p<0.0001)$. As expected, CBM-derived material properties globally stiffened the FE models. This caused model predictions to be mirrored from a slight strain overestimation in reference models, (pooled data slope 1.17) to a slight underestimation in CBM models, (slope 0.83, Fig. 3). CBM-Opt models instead achieved an almost ideal slope value of 1.01 (not significantly different from unity at $p=0.30$, while CBM and reference models had $\mathrm{p}<0.0001$ ).

\subsubsection{Femoral neck data}

When limiting the analysis to strain data from the femoral neck (Table 2 and Fig. 4, top left), where the effect of explicit cortical thickness and density modelling should be more evident, we observed a generalised improvement of accuracy indicators for CBM models. In particular, with respect to reference models:

- RMSE was almost halved ( $-44 \%$ in pooled data, $-30 \%$ in stance, and $-46 \%$ in fall)
- 95th percentiles of the FE strain error distributions were reduced by $43 \%$ (396 vs. $700 \mu \varepsilon$ ) in pooled data, by $39 \%$ (228 vs. $371 \mu \varepsilon$ ) in stance, and by $42 \%$ ( 455 vs. $784 \mu \varepsilon$ ) in fall.

- slopes of CBM models regressions were not only significantly different from those of reference models, but also closer to the ideal value of one (1.30 in reference model vs. 0.91 in CBM model), although statistical equivalence to one was not achieved due to high correlation.

Conversely, we observed a slight worsening of some CBM-Opt models indicators (Table 2 and Fig. 4, top right), and in particular of the slope of the regression line, that indicated an overall slight overestimation of strains. Although closer to unity than the reference models $(p<0.0001)$, it was no more statistically indifferent to the ideal value of one $(\mathrm{p}<0.0001)$.

\subsubsection{Strain gauge data}

The reduction observed in Peak errors, with the maximum absolute error going from $82.6 \%$ to $33.0 \%$ of the maximum measured strain value (58.6\% for CBM-Opt), led us to further process the data to report local changes in the strain predictions, by each single strain gauge location. Full strain gauge-specific plots for CBM, CBM-Opt and reference models are reported as Supplementary material (Strain Gauge Data Slides). We here describe general trends for CBM (considerations for CBM-Opt are qualitatively the same for almost all locations, with reduced differences with respect to reference data):

- at five (out of 12) locations, all in the femoral neck and head-neck junction (inferior, anterior and superior aspect of the femoral neck; anterior and superior aspect of the head-neck junction) we observed clear improvements in strain prediction accuracy, with: (i) elimination of almost all peak errors due to strain underestimation (thus likely to PVE), (ii) notable RMSE reduction, and (iii) acceptable model over-stiffening where present; we report the case of anterior neck location (Fig. 4, right), where CBM model improvements are manifest.

- at five locations, mostly in the metaphysis, (anterior and lateral aspect of the metaphysis, posterior aspect of the neck, medial aspect of the head-neck junction) error metrics were similar for reference and CBM models. In fact, while systematic strain underestimations were resolved, over-stiffening of CBM models caused symmetrical overestimations.

- at two locations (medial aspect of the metaphysis, and posterior aspect of the head-neck junction) RMSE indeed increased, although slightly, in the CBM models, likely due to the cited effect of model over-stiffening. Also at one of these two locations, however, we could observe a CBM effect in peak error reduction, by the elimination of severe strain underestimation (Supplementary material Strain Gauge Data Slides, PH strain gauge). 

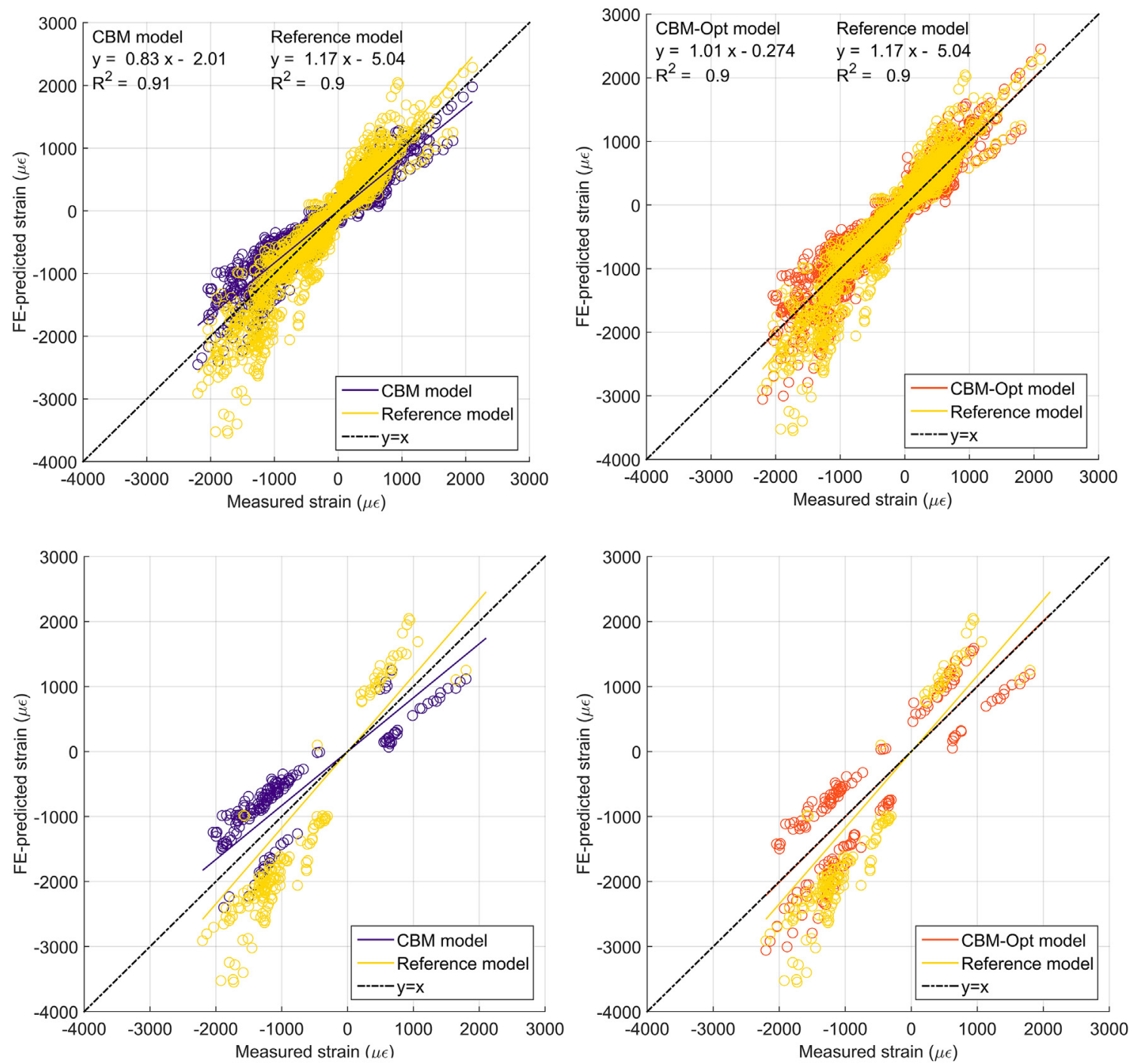

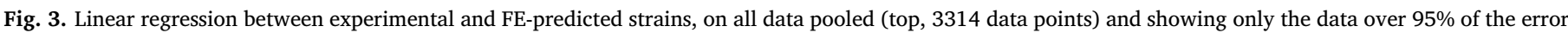
distribution (bottom, 166 data points).

\subsection{Failure load and failure location}

CBM models generally led to overestimate the failure load, with a greater overestimation in sideways fall loading condition. The error in failure load prediction was always higher for CBM models, with mean error of $64 \%$, four times that of reference models. In only one case (specimen \#5, stance) CBM improved the prediction with respect to the reference model. Linear regression for CBM models had a $\mathrm{R}^{2}$ of 0.79 , lower than that of reference models (0.89), producing an increase of $31 \%$ in SEE (1267 $\mathrm{N}$ vs. $880 \mathrm{~N}$ ). The regression line of CBM models appears offset with respect to that of reference models, due to an almost unchanged slope (CBM 0.73 vs. reference 0.72 ) and a consistent increase in the intercept (Fig. 5).

CBM-opt models showed a less marked overestimation of failure load, but the mean absolute error on prediction remained high $(42 \%$, more than twice that of reference models). Moreover, regression

Table 2

Strain accuracy indicators at the femoral neck level for CBM, CBM-Opt and reference models.

\begin{tabular}{|c|c|c|c|c|c|c|c|c|c|}
\hline \multirow[t]{2}{*}{ Strain accuracy indicators } & \multicolumn{3}{|l|}{ Pooled } & \multicolumn{3}{|l|}{ Stance } & \multicolumn{3}{|c|}{ Sideways fall } \\
\hline & CBM & Ref. & CBM-Opt & CBM & Ref. & CBM-Opt & CBM & Ref & CBM-Opt \\
\hline $\mathrm{R}^{2}$ & 0.93 & 0.92 & 0.93 & 0.93 & 0.91 & 0.93 & 0.93 & 0.93 & 0.93 \\
\hline Slope & 0.91 & 1.30 & 1.15 & 0.94 & 1.14 & 1.20 & 0.91 & 1.33 & 1.14 \\
\hline Intercept $(\mu \varepsilon)$ & 5 & 13 & 14 & -8 & 11 & -8 & 13 & 16 & 27 \\
\hline $\operatorname{SEE}(\mu \varepsilon)$ & 159 & 235 & 195 & 112 & 149 & 143 & 182 & 268 & 219 \\
\hline RMSE $(\mu \varepsilon)$ & 170 & 302 & 216 & 112 & 161 & 168 & 197 & 362 & 240 \\
\hline RMSE \% & 7.7 & 13.7 & 9.8 & 7.0 & 10.0 & 10.4 & 8.9 & 16.4 & 10.9 \\
\hline Max.err $(\mu \varepsilon)$ & 727 & 1819 & 1291 & 408 & 775 & 712 & 727 & 1819 & 1291 \\
\hline Max.err \% & 33.0 & 82.6 & 58.6 & 25.4 & 48.3 & 44.3 & 33.0 & 82.6 & 58.6 \\
\hline
\end{tabular}



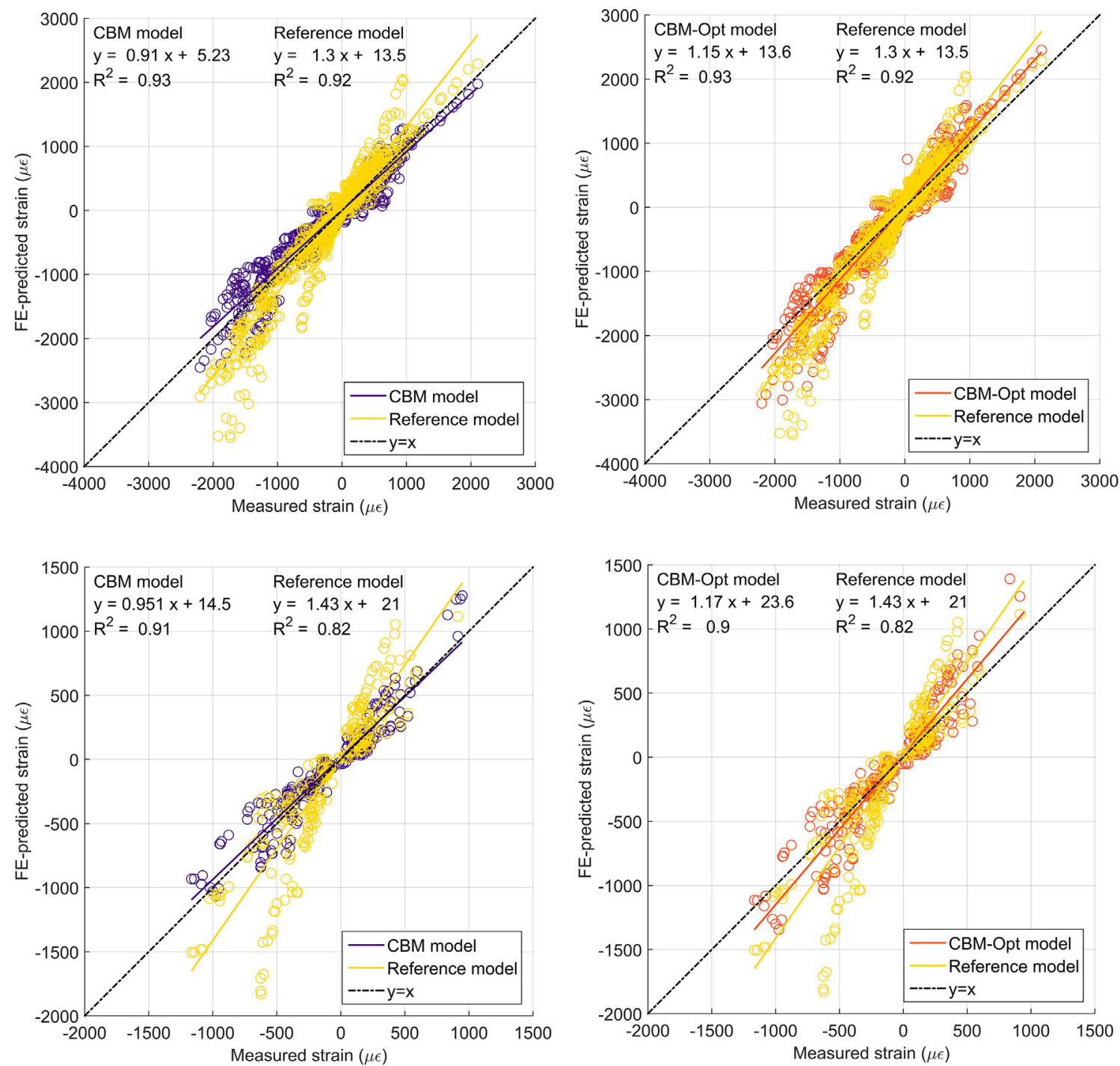

Fig. 4. Linear regression between experimental and FE-predicted strains at the femoral neck (top, 1082 data points) and at a single location (bottom), which was error-prone in reference models (strain gauge placed in the anterior femoral neck aspect, 282 data points).

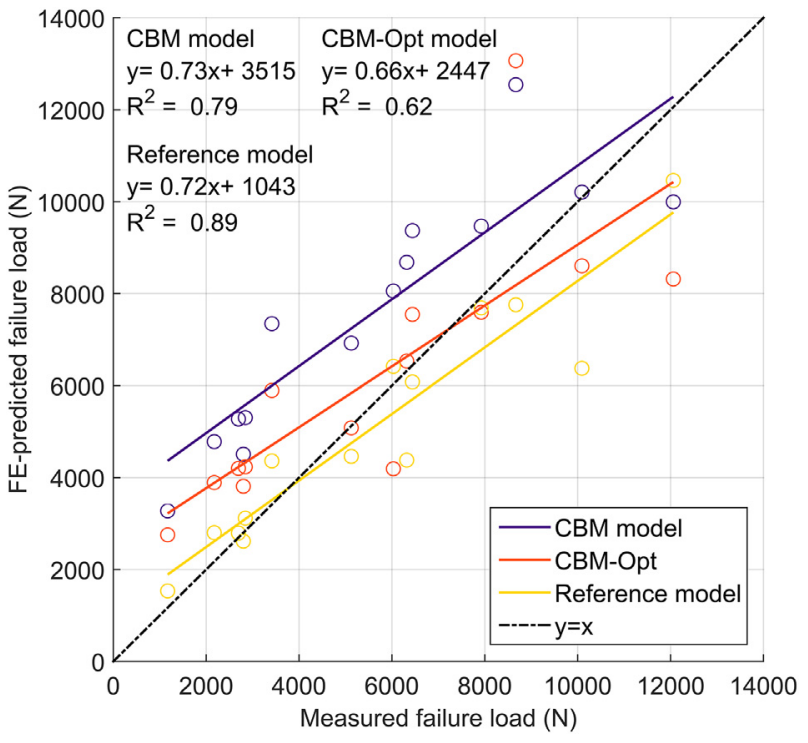

Fig. 5. Linear regression between experimental and FE failure loads for reference, CBM and CBM-Opt models. metrics were further degraded for CBM-Opt $\left(\mathrm{R}^{2}=0.62\right)$. Specimenwise strength estimates for CBM, CBM-Opt and reference models are reported together with specimen details in Supplementary material Table I.

In terms of failure location, the distance between the failed node predicted by the CBM models and that predicted by the reference models was on average $5 \mathrm{~mm}$, with the minimum and the maximum distances equal to $1 \mathrm{~mm}$ (specimen \#4) and $16 \mathrm{~mm}$ (specimen \#9). However, when categorising the experimentally observed failures into cervical, mid-neck and base-neck, like originally done for the reference models [32], in no cases did CBM models suggest a change in fracture category (Fig. 6). And even the maximum $16 \mathrm{~mm}$ difference (specimen \#9) led to an unchanged classification of failure location prediction. Almost identical observations apply to CBM-Opt failure location results, which did not differ more than a few mm from those of CBM and produced no changes in classification (Fig. 6).

\section{Discussion}

The inclusion of explicit modelling of the cortical bone compartment, based on subject specific estimates of cortical bone thickness and density, clearly improved finite element estimates of proximal femoral strains in the elastic range, both under stance- and fall-like loading 


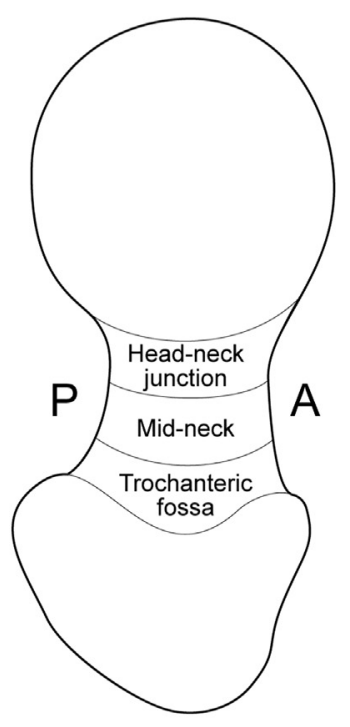

CBM
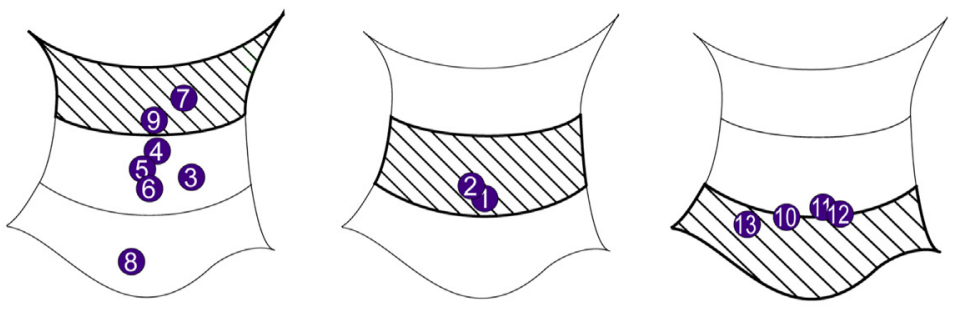

Experimental failure location
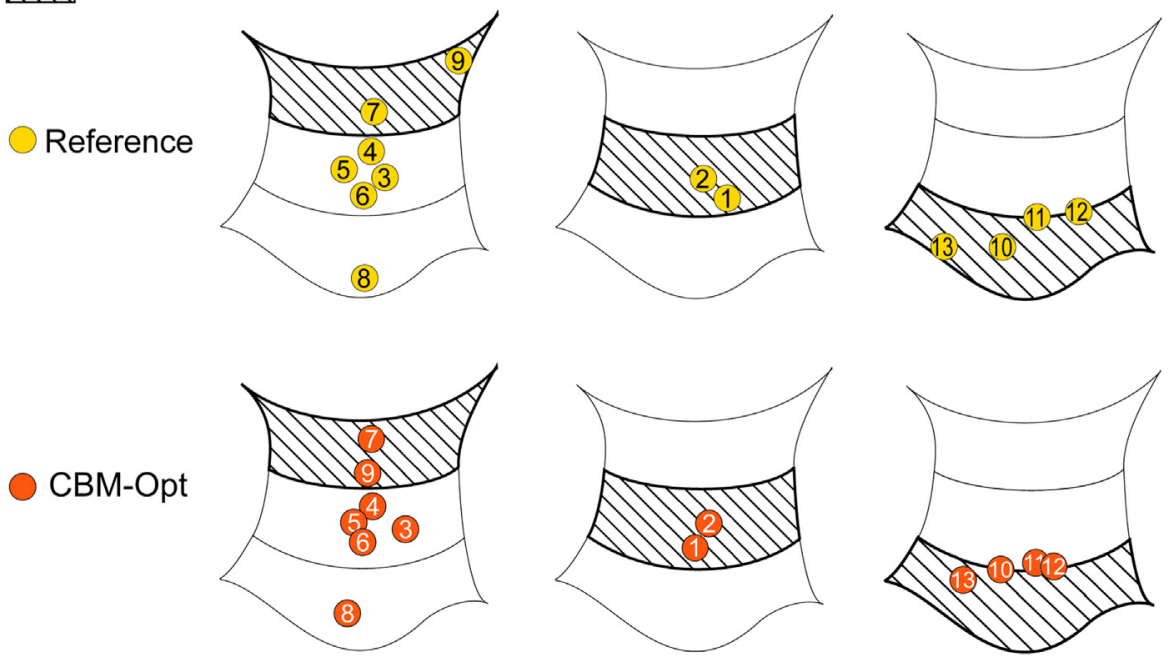

Fig. 6. Schematization of experimentally observed vs. predicted location of fracture initiation in CBM (top), reference (middle) and CBM-Opt models (bottom). Nodewise prediction of failure location is reported for each specimen (specimen number indicated in the circles). Specimen \#14 is not shown because fracture location could not be accurately detected from high-speed videos taken during the experimental test.

conditions, but not strength estimates.

\subsection{Strain accuracy}

Strain results confirmed that a straightforward implementation of CBM-derived cortical thickness and density information, featuring the explicit modelling of the cortical layer but without changes in material mapping procedures with respect to reference model can effectively counteract the FE model inaccuracies due to PVE, by providing more consistent local estimates of cortical bone elasticity. In the femoral neck, where PVE is manifest, we observed a marked improvement of all error metrics, and of the slope of the regression line, indicating femoral neck strains in CBM models were no more overestimated, as often happened in reference models. This behaviour was most evident at strain gauge sites of known thin cortex (like the anterior and superior aspects of the femoral neck and of the head-neck junction), where PVErelated FE inaccuracies had already been spotted [15]. There, all strain accuracy indicators were improved, increasing determination coefficient (meaning a better modelling of the physics), and halving (at least) errors.

The reduction of PVE-related strain errors in the proximal epiphysis was partially compensated by a slight worsening of CBM model accuracy compared to reference at metaphyseal strain gauge sites. CBM models tended to underestimate metaphyseal strains, likely due to model over-stiffening. This may seem contradictory as the accuracy of thickness estimation in reference models should be larger in thick metaphyseal cortices. However, reference models still suffer from some PVE, so that the maximum Young's modulus is found not in the outermost finite elements but in the first inner layer.

In CBM models, on the contrary, the highest modulus is found in the outermost elements.

The generalised, although slight overstiffening of CBM models (see overall regression slope, Fig. 2 and Table 2) could not be resolved by applying an upper threshold to the Young's moduli of the trabecular compartment to avoid possible double-counting of cortical-like densities already accounted for in the cortical compartment (see Appendix 1, Tables A2, A3) for the negligible validation changes induced by CBMTb.Limit, despite a lot of trabecular elements had been filtered. This finding indirectly supports, for the elastic field, the biomechanical predominance of cortical bone stated by others in terms of strength $[8,9]$. Further developments were thus called in cortical bone properties mapping. We systematically explored them through the careful choice of a cortical-specific (CBM-Cort models) and of an optimised density-elasticity relationship (CBM-Opt).

Contrarily to what expected, we reported very subtle validation improvements for CBM-Cort models (Appendix 1, Tables A2, A3), so that we stopped this exploration after two pilot specimens. It is unlikely that large errors affected the chosen density-elasticity relationship [35], as it was derived from state of the art experimental techniques focused to minimise random and systematic errors in the determination of the elastic modulus [36]. To explain the remaining discrepancies: (i) we hypothesise that future modelling of cortical bone orthotropy (or at least transverse isotropy, [50]) could improve estimate of strains developing transverse to the principal direction; however, subject specific orthotropy information is currently not accessible; (ii) we explored the adoption of an optimised density-elasticity relationships, derived from an independent, large dataset (CBM-Opt).

CBM-Opt achieved an impressive unitary value of the regression slope in pooled data, but inter-specimen variations in slope remained notable and similar to reference and CBM models (see specimen-wise strain accuracy metrics in Supplementary material, Table III). The improvement of the overall CBM-Opt slope came at the cost of error metrics, which although reduced with respect to reference models were worse than in CBM. Also, CBM-opt, like CBM, could not improve the overall $R^{2}$. These results suggest that changes in universal densityelasticity relationship bring unavoidable trade-offs, and that either 
additional physical mechanisms should be accounted for, and/or limits of CT data in providing subject-specific bone elasticity information should be acknowledged [26]. We believe that a lot remains to be clarified about intra and inter-subject variability of density-elasticity relationships, but our experiment cannot help. This knowledge should come from experimental and numerical studies accessing also lower scales of material organization to explain the differences found.

Despite these limitations, which appear inherent to current CTbased FE modelling, the sum of local findings induced in CBM pooled data (and to a lower extent also in CBM-Opt) a marked reduction of peak errors, which were mostly occurring at sites of thin cortical shell, and a generalised reduction of all error metrics. This is a clear overall improvement brought by subject-specific modelling of cortical thickness and density. The reliability of our strain accuracy results is corroborated by the size of the experimental dataset. With 10 femurs instrumented with 12 triaxial strain gauges and tested in different loading configurations for a total of 3314 measurements, it is to our knowledge the largest femoral dataset available in which bone strains were measured. Conversely, our experimental setup was not well devised to achieve validation of bone stiffness under load. In fact, only load-displacement curves from the loading machine, measuring the whole actuator-load cell stiffness chain (made of bearings, cement pots and interfaces, thus not reliable for bone stiffness measurement) were available for the majority of specimens. Prospectively an extension to full-field measurements [51] would be welcome, to fully assess the elastic response (local surface strains and overall bone stiffness) of bone specimens.

The improved strain accuracy obtained with CBM models leads us to promote subject-specific modelling of cortical bone thickness and density in all cases where accurate estimates of elastic bone strains are needed. We are thinking in particular of the estimate of physiological and exercise-induced strains. It is clear that exercise [52], and especially impact exercise [53] contrasts bone loss, with evident benefit at cortical sites [54]. FE simulations may help designing targeted impact exercises to elicit bone-promoting, but not harmful strains [55]. In this context, CBM models would improve accuracy and safety of the exercise design, by providing improved strain prediction at focal areas of the proximal femur.

\subsection{Strength accuracy}

Failure load predictions of CBM and CBM-Opt models did not reflect the improvements in strain prediction accuracy, although the failure criterion was strain-based. Failure load was systematically, and greatly overestimated by CBM models. Failure load overestimation was even more marked for specimens tested in fall, where structural collapse, although possibly triggered by a small amount of trabecular crushing $[56,57]$, is often made evident first by a compressive failure of the superior neck cortex [58] i.e. exactly where CBM models showed the highest improvements in strain accuracy. The $-10,400$ microstrain compressive limit we adopted may be questioned because obtained on trabecular bone specimens [49]. We thus attempted a post-processing of our results by adopting a reduced compressive limit of $-8600 \mathrm{mi}-$ crostrain after a cortical study [39], already used in other fracture studies [59]. However, this modification had almost no effect but a change in the regression line intercept, meaning CBM models need exploration of more refined failure criteria. Despite a less marked overestimation of individual failure loads with respect to CBM, the overall performance of CBM-Opt models in estimating strength was even worse, as the $\mathrm{R}^{2}$ (a key parameter for the use of estimated strength as fracture risk classifier in clinical studies) significantly degraded. We neither reported significant changes in the estimate of failure location for CBM or CBM-Opt vs. reference models. This confirms that reference models already captured rather well the region at risk for sub-capital and mid-neck fractures (for a full report see [32]), and suggest changing the simulated failure mechanism (e.g. considering elastic instability) to attempt a correct identification of base-neck fractures, all occurred in sideways fall loading.

By testing strength estimates of both CBM and CBM-Opt models we accumulated evidence that the simple asymmetric principal strain criterion yielding good results in the reference models, although not optimised but extracted from independent, lower scale experiments and considerations, is not able to give equally good results when used on models that are more accurate in estimating elastic strains at focal sites of fracture [21]. This may suggest that a sort of tradeoff between strain inaccuracies and simplified fracture criterion had been reached in the reference models. The unsuccessful attempt to reduce the compressive limit seems to confirm this, but a full testing of this hypothesis is left to future studies, possibly on larger samples. Despite our falsification, on CBM-derived models, of the simple principal strain criterion, we believe it still represents a good standard, since reference models were successfully used in-vivo to classify fracture cases and controls [60]. We hope our work may stimulate the development of new, biofidelic criteria from strain-accurate CBM models.

\subsection{Literature comparison, limitations and outlook}

In predicting strains, the present study extends the positive findings of previous works that attempted to compensate for PVE in CT-based FE modelling, through node-based material mapping refinements alone ([16], at the femur) or combined with a CT deconvolution algorithm that shares some premises with CBM but actually produces "deblurred CT images" from which FE models are built ([61], maxillofacial skeleton). In a past study, we used this CT deblurring to build FE models of the proximal femur, on the same experimental data here presented [18]. While both CBM and deblurring approaches led to improved strain accuracy, error reductions were more evident in CBM models, especially for peak errors, and at femoral neck locations, because of better PVE reduction at the anterior and superior femoral neck aspects. A comparison of the performance of CBM, CBM-Opt, reference, and deblurred models is reported as Supplementary material - Table II. The more refined CT deconvolution algorithm adopted to obtain CBM estimates, accounting for out of plane blurring, may have contributed to this improved performance of CBM models, but we relate the improvement mainly to the modification of FE topology by definition of a cortical volume and assignment of dedicated properties.

An implementation of CBM into FE including experimental validation has been very recently published [22]. Apparently their results seem to partially contradict ours, as reported strain accuracies did not improve over manually generated FE models. However, in their work CBM was used mainly to achieve a fully automatic segmentation and modelling procedure, with a possible trade off with accuracy. Moreover, it is not clear if and how they treated density information from CBM. Other algorithms that produce validated subject specific estimates of cortical thickness have been recently published and may be candidate to be included in FE models. However, regardless of the implementation (from image deconvolution approaches similar in concept to CBM $[62,63]$, to statistical models that swiftly produce FEfriendly surfaces [64]) they do not provide local cortical density estimates, as they all assume for density a fully mineralised condition. Knowledge of cortical thickness can be enough to improve FE modelling of the overall bone structural behaviour and simulated stress, but local strains are highly influenced by the elastic modulus, in turn related to density estimates. This theoretical statement was corroborated by a preliminary work we did on two femurs [65]: modelling only cortical thickness with the CBMv1 algorithm [66], and adopting a uniform cortical elastic modulus of $18 \mathrm{GPa}$ brought to severe global over-stiffening. The CBM models here described instead incorporated into finite elements, for the first time to our knowledge, subject specific estimates of both thickness and density of cortical bone from clinical CT data.

We are aware that cortical bone has a "challenging geography" [1], of which we here capture only two features, with several limitations 
associated to the image source and the modelling process:

- cortical thickness and density estimates may be inaccurate, to a certain extent. Density estimates are particularly prone to overestimations below true radiological densities of $800 \mathrm{mg} / \mathrm{cm}^{3}$ ([19], checked against HRpQCT), which are, however, rarely found. We acknowledge this is an unavoidable compromise to cortical estimates from CT, and may account for part of the observed CBM models over-stiffening.

- cortical density is modelled as constant over thickness. Doing so we could neither capture the progressive trabecularization from the endosteal cortical boundary, typical of aging [67], nor other diseasedependent emergencies of cortical macro-pores. However, our models can indirectly discriminate, within the accuracy of densitometric calibration, the lumped effect of an abnormal cortical porosity and its variations. An extension of CBM that considers a variable density over the cortex thickness has been recently proposed [68]. Its inclusion in FE models may help reducing the over-stiffening effect observed in some femoral regions. However, this would need either a multi-layer modelling of the bone cortex, or an element-variable elastic modulus assignment strategy, which were out of the scope of the present study.

- we used a threshold to discard local failures of the optimization algorithm. Although thresholding was limited to few percentages of elements, an interpolation scheme from surrounding elements may be more appropriate when mapping materials to FE models.

While it could be worth exploring the above proposed modelling refinements, we believe the priority for the technical development of our modelling procedure is to tackle strength predictions by enriching CBM models with appropriate fracture criteria.

In summary:

- we proposed for the first time FE models of the proximal femur that incorporate local, subject-specific estimates of cortical thickness and density from clinical CT data

- we corroborated our main research hypothesis i.e. that subjectspecific modelling of cortical bone improves bone strain estimates in the elastic field of deformations; CBM-derived models can thus be used to define/improve estimates of bone strains elicited by exercise

- we showed that changes in the cortical density-elasticity relationship, according to a state of the art experiment or to independently optimised parameters could not dramatically alter the results

- we failed to accurately predict failure characteristics with CBMderived models. We are however convinced that CBM-derived models may provide more trustworthy strain data to develop biofidelic failure criteria.

Supplementary data to this article can be found online at https:// doi.org/10.1016/j.bone.2020.115348.

\section{CRediT authorship contribution statement}

Enrico Schileo: Conceptualization, Methodology, Formal analysis, Funding acquisition, Supervision, Writing - original draft. Jonathan Pitocchi: Methodology, Formal analysis, Writing - original draft. Cristina Falcinelli: Methodology, Formal analysis, Visualization, Writing - original draft. Fulvia Taddei: Conceptualization, Resources, Funding acquisition, Supervision, Visualization, Writing - review \& editing.

\section{Acknowledgements}

This work was supported by the Italian Ministry of Health (qSINS project, grant number: RF-2016-02364359) and by the Marie Skłodowska-Curie ITN CuraBone (722535).
We would like to thank Graham Treece (University of Cambridge) for his help in using the Stradwin software, and Luigi Lena (IRCSS Istituto Ortopedico Rizzoli) for the artwork.

\section{References}

[1] R. Zebaze, E. Seeman, Cortical bone: a challenging geography, J. Bone Miner. Res. 30 (2015) 24-29, https://doi.org/10.1002/jbmr.2419.

[2] J. Reeve, N. Loveridge, The fragile elderly hip: mechanisms associated with agerelated loss of strength and toughness, Bone 61 (2014) 138-148, https://doi.org/ 10.1016/j.bone.2013.12.034.

[3] J. Power, N. Loveridge, H. Kröger, M. Parker, J. Reeve, Femoral neck cortical bone in female and male hip fracture cases: differential contrasts in cortical width and sub-periosteal porosity in 112 cases and controls, Bone 114 (2018) 81-89, https:// doi.org/10.1016/j.bone.2018.05.027.

[4] K.E. Poole, G.M. Treece, A.H. Gee, J.P. Brown, M.R. McClung, A. Wang, C. Libanati, Denosumab rapidly increases cortical bone in key locations of the femur: a 3D bone mapping study in women with osteoporosis: denosumab increases femoral cortical bone, J. Bone Miner. Res. 30 (2015) 46-54, https://doi.org/10.1002/jbmr.2325.

[5] P.M. Mayhew, C.D. Thomas, J.G. Clement, N. Loveridge, T.J. Beck, W. Bonfield, C.J. Burgoyne, J. Reeve, Relation between age, femoral neck cortical stability, and hip fracture risk, Lancet 366 (2005) 129-135, https://doi.org/10.1016/S01406736(05)66870-5.

[6] F. Johannesdottir, E. Thrall, J. Muller, T.M. Keaveny, D.L. Kopperdahl, M.L. Bouxsein, Comparison of non-invasive assessments of strength of the proximal femur, Bone 105 (2017) 93-102, https://doi.org/10.1016/j.bone.2017.07.023.

[7] S.L. Manske, T. Liu-Ambrose, D.M.L. Cooper, S. Kontulainen, P. Guy, B.B. Forster, H.A. McKay, Cortical and trabecular bone in the femoral neck both contribute to proximal femur failure load prediction, Osteoporos. Int. 20 (2009) 445-453, https://doi.org/10.1007/s00198-008-0675-2.

[8] J.E.M. Koivumäki, J. Thevenot, P. Pulkkinen, V. Kuhn, T.M. Link, F. Eckstein, T. Jämsä, Cortical bone finite element models in the estimation of experimentally measured failure loads in the proximal femur, Bone 51 (2012) 737-740, https:// doi.org/10.1016/j.bone.2012.06.026.

[9] G. Holzer, G. von Skrbensky, L.A. Holzer, W. Pichl, Hip fractures and the contribution of cortical versus trabecular bone to femoral neck strength, J. Bone Miner. Res. 24 (2009) 468-474, https://doi.org/10.1359/jbmr.081108.

[10] S. Nawathe, B.P. Nguyen, N. Barzanian, H. Akhlaghpour, M.L. Bouxsein, T.M. Keaveny, Cortical and trabecular load sharing in the human femoral neck, J. Biomech. 48 (2015) 816-822, https://doi.org/10.1016/j.jbiomech.2014.12.022.

[11] M. Bessho, I. Ohnishi, J. Matsuyama, T. Matsumoto, K. Imai, K. Nakamura, Prediction of strength and strain of the proximal femur by a CT-based finite element method, J. Biomech. 40 (2007) 1745-1753, https://doi.org/10.1016/j.jbiomech. 2006.08.003.

[12] J. Rittweger, I. Michaelis, M. Giehl, P. Wüsecke, D. Felsenberg, Adjusting for the Partial Volume Effect in Cortical Bone Analyses of pQCT Images, (2004), p. 6.

[13] A. Maloul, J. Fialkov, C. Whyne, The impact of voxel size-based inaccuracies on the mechanical behavior of thin bone structures, Ann. Biomed. Eng. 39 (2011) 1092-1100, https://doi.org/10.1007/s10439-010-0215-z.

[14] E. Peleg, R. Herblum, M. Beek, L. Joskowicz, M. Liebergall, R. Mosheiff, C. Whyne, Can a partial volume edge effect reduction algorithm improve the repeatability of subject-specific finite element models of femurs obtained from CT data? Computer Methods in Biomechanics and Biomedical Engineering 17 (2014) 204-209, https:// doi.org/10.1080/10255842.2012.673595.

[15] L. Grassi, E. Schileo, F. Taddei, L. Zani, M. Juszczyk, L. Cristofolini, M. Viceconti, Accuracy of finite element predictions in sideways load configurations for the proximal human femur, J. Biomech. 45 (2012) 394-399, https://doi.org/10.1016/ j.jbiomech.2011.10.019.

[16] B. Helgason, F. Taddei, H. Pálsson, E. Schileo, L. Cristofolini, M. Viceconti, S. Brynjólfsson, A modified method for assigning material properties to FE models of bones, Med. Eng. Phys. 30 (2008) 444-453, https://doi.org/10.1016/j. medengphy.2007.05.006.

[17] T.D. Szwedowski, J. Fialkov, C.M. Whyne, Sensitivity analysis of a validated subject-specific finite element model of the human craniofacial skeleton, Proc. Inst. Mech. Eng. H J. Eng. Med. 225 (2011) 58-67, https://doi.org/10.1243/ 09544119 JEIM786.

[18] C. Falcinelli, E. Schileo, A. Pakdel, C. Whyne, L. Cristofolini, F. Taddei, Can CT image deblurring improve finite element predictions at the proximal femur? J. Mech. Behav. Biomed. Mater. 63 (2016) 337-351, https://doi.org/10.1016/j. jmbbm.2016.07.004.

[19] G.M. Treece, A.H. Gee, Independent measurement of femoral cortical thickness and cortical bone density using clinical CT, Med. Image Anal. 20 (2015) 249-264, https://doi.org/10.1016/j.media.2014.11.012.

[20] G.M. Treece, A.H. Gee, C. Tonkin, S.K. Ewing, P.M. Cawthon, D.M. Black, K.E. Poole, for the Osteoporotic Fractures in Men (MrOS) Study, Predicting hip fracture type with cortical bone mapping (CBM) in The osteoporotic fractures in men (MrOS) study: predicting hip fracture type with CBM in the MrOS study, J. Bone Miner. Res. 30 (2015) 2067-2077, https://doi.org/10.1002/jbmr.2552.

[21] K.E.S. Poole, L. Skingle, A.H. Gee, T.D. Turmezei, F. Johannesdottir, K. Blesic, C. Rose, M. Vindlacheruvu, S. Donell, J. Vaculik, P. Dungl, M. Horak, J.J. Stepan, J. Reeve, G.M. Treece, Focal osteoporosis defects play a key role in hip fracture, Bone 94 (2017) 124-134, https://doi.org/10.1016/j.bone.2016.10.020.

[22] S.P. Väänänen, L. Grassi, M.S. Venäläinen, H. Matikka, Y. Zheng, J.S. Jurvelin, $\mathrm{H}$. Isaksson, Automated segmentation of cortical and trabecular bone to generate 
finite element models for femoral bone mechanics, Med. Eng. Phys. 70 (2019) 19-28, https://doi.org/10.1016/j.medengphy.2019.06.015.

[23] S.L. Schoell, A.A. Weaver, D.P. Beavers, L. Lenchik, A.P. Marsh, W.J. Rejeski, J.D. Stitzel, K.M. Beavers, Development of subject-specific proximal femur finite element models of older adults with obesity to evaluate the effects of weight loss on bone strength, Journal of Osteoporosis and Physical Activity 6 (2018) 213, https:// doi.org/10.4172/2329-9509.1000213.

[24] E.F. Morgan, H.H. Bayraktar, T.M. Keaveny, Trabecular bone modulus-density relationships depend on anatomic site, J. Biomech. 36 (2003) 897-904, https://doi. org/10.1016/S0021-9290(03)00071-X.

[25] S. Eberle, M. Göttlinger, P. Augat, Individual density-elasticity relationships improve accuracy of subject-specific finite element models of human femurs, J. Biomech. 46 (2013) 2152-2157, https://doi.org/10.1016/j.jbiomech.2013.06.035.

[26] H. Wille, E. Rank, Z. Yosibash, Prediction of the mechanical response of the femur with uncertain elastic properties, J. Biomech. 45 (2012) 1140-1148, https://doi. org/10.1016/j.jbiomech.2012.02.006.

[27] A. Rezaei, K.D. Carlson, H. Giambini, S. Javid, D. Dragomir-Daescu, Optimizing accuracy of proximal femur elastic modulus equations, Ann. Biomed. Eng. 47 (2019) 1391-1399, https://doi.org/10.1007/s10439-019-02238-9.

[28] L. Cristofolini, M. Juszczyk, F. Taddei, M. Viceconti, Strain distribution in the proximal human femoral metaphysis, Proc Inst Mech Eng H 223 (2009) 273-288, https://doi.org/10.1243/09544119JEIM497.

[29] L. Zani, P. Erani, L. Grassi, F. Taddei, L. Cristofolini, Strain distribution in the proximal human femur during in vitro simulated sideways fall, J. Biomech. 48 (2015) 2130-2143, https://doi.org/10.1016/j.jbiomech.2015.02.022.

[30] E. Schileo, F. Taddei, A. Malandrino, L. Cristofolini, M. Viceconti, Subject-specific finite element models can accurately predict strain levels in long bones, J. Biomech. 40 (2007) 2982-2989, https://doi.org/10.1016/j.jbiomech.2007.02.010.

[31] E. Schileo, E. Dall'Ara, F. Taddei, A. Malandrino, T. Schotkamp, M. Baleani, M. Viceconti, An accurate estimation of bone density improves the accuracy of subject-specific finite element models, J. Biomech. 41 (2008) 2483-2491, https:// doi.org/10.1016/j.jbiomech.2008.05.017.

[32] E. Schileo, L. Balistreri, L. Grassi, L. Cristofolini, F. Taddei, To what extent can linear finite element models of human femora predict failure under stance and fall loading configurations? J. Biomech. 47 (2014) 3531-3538, https://doi.org/10. 1016/j.jbiomech.2014.08.024.

[33] F. Taddei, A. Pancanti, M. Viceconti, An improved method for the automatic mapping of computed tomography numbers onto finite element models, Med. Eng. Phys. 26 (2004) 61-69, https://doi.org/10.1016/S1350-4533(03)00138-3.

[34] F. Taddei, E. Schileo, B. Helgason, L. Cristofolini, M. Viceconti, The material mapping strategy influences the accuracy of CT-based finite element models of bones: an evaluation against experimental measurements, Med. Eng. Phys. 29 (2007) 973-979, https://doi.org/10.1016/j.medengphy.2006.10.014.

[35] C. Öhman, M. Baleani, C. Pani, F. Taddei, M. Alberghini, M. Viceconti, M. Manfrini, Compressive behaviour of child and adult cortical bone, Bone 49 (2011) 769-776, https://doi.org/10.1016/j.bone.2011.06.035.

[36] B. Helgason, E. Perilli, E. Schileo, F. Taddei, S. Brynjólfsson, M. Viceconti, Mathematical relationships between bone density and mechanical properties: a literature review, Clin. Biomech. 23 (2008) 135-146, https://doi.org/10.1016/j. clinbiomech.2007.08.024.

[37] B.B. Seedhom, E. Berry, A.E. Ostell, M. Cuppone, The longitudinal Young's modulus of cortical bone in the midshaft of human femur and its correlation with CT scanning data, Calcif. Tissue Int. 74 (2004) 302-309, https://doi.org/10.1007/s00223002-2123-1.

[38] L. Duchemin, V. Bousson, C. Raossanaly, C. Bergot, J.D. Laredo, W. Skalli, D. Mitton, Prediction of mechanical properties of cortical bone by quantitative computed tomography, Med. Eng. Phys. 30 (2008) 321-328, https://doi.org/10. 1016/j.medengphy.2007.04.008.

[39] T.S. Kaneko, M.R. Pejcic, J. Tehranzadeh, J.H. Keyak, Relationships between material properties and CT scan data of cortical bone with and without metastatic lesions, Med. Eng. Phys. 25 (2003) 445-454, https://doi.org/10.1016/S13504533(03)00030-4.

[40] J.C. Lotz, T.N. Gerhart, W.C. Hayes, Mechanical properties of metaphyseal bone in the proximal femur, J. Biomech. 24 (1991) 317-329, https://doi.org/10.1016/ 0021-9290(91)90350-v.

[41] S. Bernard, J. Schneider, P. Varga, P. Laugier, K. Raum, Q. Grimal, Elasticity-density and viscoelasticity-density relationships at the tibia mid-diaphysis assessed from resonant ultrasound spectroscopy measurements, Biomech. Model. Mechanobiol. 15 (2016) 97-109, https://doi.org/10.1007/s10237-0150689-6.

[42] D.R. Carter, W.C. Hayes, The compressive behavior of bone as a two-phase porous structure, J. Bone Joint Surg. Am. 59 (1977) 954-962.

[43] T.S. Keller, Predicting the compressive mechanical behavior of bone, J. Biomech. 27 (1994) 1159-1168, https://doi.org/10.1016/0021-9290(94)90056-6.

[44] J.Y. Rho, M.C. Hobatho, R.B. Ashman, Relations of mechanical properties to density and CT numbers in human bone, Med. Eng. Phys. 17 (1995) 347-355, https://doi. org/10.1016/1350-4533(95)97314-F.

[45] S.M. Snyder, E. Schneider, Estimation of mechanical properties of cortical bone by computed tomography, J. Orthop. Res. 9 (1991) 422-431, https://doi.org/10. 1002/jor.1100090315.

[46] K.H.J. Groenen, T. Bitter, T.C.G. van Veluwen, Y.M. van der Linden, N. Verdonschot, E. Tanck, D. Janssen, Case-specific non-linear finite element models to predict failure behavior in two functional spinal units: Fe to predict vertebral failure, J. Orthop. Res. (2018), https://doi.org/10.1002/jor.24117.

[47] M. Tuncer, U.N. Hansen, A.A. Amis, Prediction of structural failure of tibial bone models under physiological loads: effect of CT density-modulus relationships, Med. Eng. Phys. 36 (2014) 991-997, https://doi.org/10.1016/j.medengphy.2014.04. 006.

[48] S.M. Nazemi, M. Amini, S.A. Kontulainen, J.S. Milner, D.W. Holdsworth, B.A. Masri, D.R. Wilson, J.D. Johnston, Optimizing finite element predictions of local subchondral bone structural stiffness using neural network-derived density-modulus relationships for proximal tibial subchondral cortical and trabecular bone, Clin. Biomech. 41 (2017) 1-8, https://doi.org/10.1016/j.clinbiomech.2016.10.012.

[49] H.H. Bayraktar, E.F. Morgan, G.L. Niebur, G.E. Morris, E.K. Wong, T.M. Keaveny, Comparison of the elastic and yield properties of human femoral trabecular and cortical bone tissue, J. Biomech. 37 (2004) 27-35, https://doi.org/10.1016/S00219290(03)00257-4.

[50] X. Neil Dong, X. Edward Guo, The dependence of transversely isotropic elasticity of human femoral cortical bone on porosity, J. Biomech. 37 (2004) 1281-1287, https://doi.org/10.1016/j.jbiomech.2003.12.011.

[51] L. Grassi, H. Isaksson, Extracting accurate strain measurements in bone mechanics: a critical review of current methods, J. Mech. Behav. Biomed. Mater. 50 (2015) 43-54, https://doi.org/10.1016/j.jmbbm.2015.06.006.

[52] T.E. Howe, B. Shea, L.J. Dawson, F. Downie, A. Murray, C. Ross, R.T. Harbour, L.M. Caldwell, G. Creed, Exercise for preventing and treating osteoporosis in postmenopausal women, Cochrane Database Syst. Rev. (2011), https://doi.org/10. 1002/14651858.CD000333.pub2.

[53] B.R. Beck, R.M. Daly, M.A.F. Singh, D.R. Taaffe, Exercise and Sports Science Australia (ESSA) position statement on exercise prescription for the prevention and management of osteoporosis, J. Sci. Med. Sport 20 (2017) 438-445, https://doi. org/10.1016/j.jsams.2016.10.001.

[54] S. Allison, J. Folland, W. Rennie, G. Summers, K. Brooke-Wavell, High impact exercise increased femoral neck bone mineral density in older men: a randomised unilateral intervention, Bone 53 (2013) 321-328, https://doi.org/10.1016/j.bone. 2012.12.045.

[55] M.E. Kersh, S. Martelli, R. Zebaze, E. Seeman, M.G. Pandy, Mechanical loading of the femoral neck in human locomotion: mechanical loading of the femoral neck in human locomotion, J. Bone Miner. Res. 33 (2018) 1999-2006, https://doi.org/10. 1002/jbmr.3529.

[56] S. Nawathe, H. Akhlaghpour, M.L. Bouxsein, T.M. Keaveny, Microstructural failure mechanisms in the human proximal femur for sideways fall loading: femoral strength micromechanics, J. Bone Miner. Res. 29 (2014) 507-515, https://doi.org/ 10.1002/jbmr.2033.

[57] H. Bahaloo, W.S. Enns-Bray, I. Fleps, O. Ariza, S. Gilchrist, R.W. Soyka, P. Guy, H. Palsson, S.J. Ferguson, P.A. Cripton, B. Helgason, On the failure initiation in the proximal human femur under simulated sideways fall, Ann. Biomed. Eng. 46 (2018) 270-283, https://doi.org/10.1007/s10439-017-1952-z.

[58] P.M. de Bakker, S.L. Manske, V. Ebacher, T.R. Oxland, P.A. Cripton, P. Guy, During sideways falls proximal femur fractures initiate in the superolateral cortex: evidence from high-speed video of simulated fractures, J. Biomech. 42 (2009) 1917-1925, https://doi.org/10.1016/j.jbiomech.2009.05.001.

[59] G. Dahan, N. Trabelsi, O. Safran, Z. Yosibash, Finite element analyses for predicting anatomical neck fractures in the proximal humerus, Clin. Biomech. 68 (2019) 114-121, https://doi.org/10.1016/j.clinbiomech.2019.05.028.

[60] C. Falcinelli, E. Schileo, L. Balistreri, F. Baruffaldi, B. Bordini, M. Viceconti, U. Albisinni, F. Ceccarelli, L. Milandri, A. Toni, F. Taddei, Multiple loading conditions analysis can improve the association between finite element bone strength estimates and proximal femur fractures: a preliminary study in elderly women, Bone 67 (2014) 71-80, https://doi.org/10.1016/j.bone.2014.06.038.

[61] A. Pakdel, J. Fialkov, C.M. Whyne, High resolution bone material property assignment yields robust subject specific finite element models of complex thin bone structures, J. Biomech. 49 (2016) 1454-1460, https://doi.org/10.1016/j.jbiomech. 2016.03.015.

[62] T. Damm, J.A. Peña, G.M. Campbell, J. Bastgen, R. Barkmann, C.-C. Glüer, Improved accuracy in the assessment of vertebral cortical thickness by quantitative computed tomography using the Iterative Convolution OptimizatioN (ICON) method, Bone 120 (2019) 194-203, https://doi.org/10.1016/j.bone.2018.08.024.

[63] O. Museyko, B. Gerner, K. Engelke, A new method to determine cortical bone thickness in CT images using a hybrid approach of parametric profile representation and local adaptive thresholds: accuracy results, PLoS One 12 (2017) e0187097, , https://doi.org/10.1371/journal.pone.0187097.

[64] V. Chandran, G. Maquer, T. Gerig, P. Zysset, M. Reyes, Supervised learning for bone shape and cortical thickness estimation from CT images for finite element analysis, Med. Image Anal. 52 (2019) 42-55, https://doi.org/10.1016/j.media.2018.11.001.

[65] J. Pitocchi, Susanna Paletti, Beatrice Cominoli, Fulvia Taddei, Enrico Schileo, Does cortical bone mapping improve FE strain prediction accuracy at the proximal femur? Proceedings, Vairo, G, Rome, 2017, pp. 91-92.

[66] G.M. Treece, K.E.S. Poole, A.H. Gee, Imaging the femoral cortex: thickness, density and mass from clinical CT, Med. Image Anal. 16 (2012) 952-965, https://doi.org/ 10.1016/j.media.2012.02.008.

[67] E. Seeman, P.D. Delmas, Bone quality - the material and structural basis of bone strength and fragility, N. Engl. J. Med. 354 (2006) 2250-2261, https://doi.org/10. 1056/NEJMra053077.

[68] R.A. Pearson, G.M. Treece, Measurement of the bone endocortical region using clinical CT, Med. Image Anal. 44 (2018) 28-40, https://doi.org/10.1016/j.media. 2017.11.006. 